\title{
Application of video glasses for sustained affective picture presentations: A comparison with video projector presentations
}

\author{
ANDREAS VON LEUPOLDT, BETTINA HARTLE-BREMERICH, and BERNHARD DAHME \\ University of Hamburg, Hamburg, Germany
}

\begin{abstract}
Video glasses have previously been reported to be an effective instrument for emotion induction or for reducing pain perception. In the present study, we assessed the application of video glasses within the affective picture viewing paradigm. Possible advantages of this new method were tested by a comparison with a commonly used video projector. Twenty-four participants viewed sustained picture series of pleasant, neutral, and unpleasant valence with video glasses and with a projector on different days. Affective report, heart rate (HR), and skin conductance level were measured. The results showed that the video glasses presentation mode is not generally superior to the projector mode. Instead, typical responses to prolonged presentations of emotional pictures were obtained across both modalities with valence-modulated pleasure ratings and with an arousal modulation for arousal ratings, skin conductance level, and HR. These results suggest that video glasses are as useful for presenting prolonged affective picture series as are projectors. Specific contexts in which the application of video glasses is preferential are discussed.
\end{abstract}

Previous studies have shown a successful application of video glasses in several areas of research. Berenson, Wiemann, and Rickert (1998) reported decreased anxiety levels in children who were distracted with this instrument from an unpleasant genital examination. Reduced anxiety and discomfort was obtained by Frere, Crout, Yorty, and McNeil (2001) in patients undergoing dental surgery while focused on a presentation provided with an audiovisual eyeglass system. Bentsen, Svensson, and Wenzel (1999) reported lower ratings for pain and unpleasantness when participants were distracted from a cold pressor test with video glasses. These results have shown that video glasses are able to strongly involve users and to help them focus their attention on presented stimuli despite parallel unpleasant stimulation, which normally demands much of our cognitive resources. A key attribute of this relatively new instrument is the impression of closeness of the material to be viewed. Furthermore, attention-distracting influences associated with laboratory environments are reduced by the limited ability to look around. This leads to a stronger sense of presence; that is, a mediated experience (e.g., viewing a film) is not perceived as being mediated by a technical device but rather is perceived as natural, immediate, real, and involving (Lombard \& Ditton, 1997).

The authors thank Thomas Ritz for his helpful comments on a previous draft of this article. The authors declare that they have no competing or financial interests in products that are referred to in this article. Reprint requests and correspondence concerning this article should be addressed to A. von Leupoldt, Psychological Institute III, University of Hamburg, Von-Melle-Park 5, 20146 Hamburg, Germany (e-mail: andreas.vonleupoldt@uni-hamburg.de).
An enhanced sense of presence produced by new presentation media, in turn, is known to increase psychophysiological responses in comparison with traditional media, but research on this topic is still in its infancy (Ravaja, 2004).

In a recent study (von Leupoldt \& Dahme, 2005), we used video glasses to present prolonged affective picture series in order to overcome line-of-sight limitations of a complex respiratory measuring cabin that allowed only a restricted view of a presentation monitor. This emerged as an effective new method for displaying slides from the International Affective Picture System (IAPS; Center for the Study of Emotion and Attention, 1999), which are typically presented via monitors or projectors (see the studies discussed below). Moreover, when we compared the results of our 2005 study with those of some of our previous studies, in which IAPS pictures were presented on screens (Ritz, Alatupa, Thöns, \& Dahme, 2002; Ritz, George, \& Dahme, 2000), we observed that participants reported higher arousal with video glasses. Furthermore, the participants reported less distraction from the complex respiratory measuring cabin than did participants in a prior study in which emotional stimuli were presented on a monitor (von Leupoldt \& Dahme, 2004). These were interesting findings with regard to typical small effect sizes in psychophysiological research (Ravaja, 2004), which typically requires emotional stimuli to be as intensive and arousing as possible (Hamm \& Vaitl, 1993).

When these facts were considered together, the question arose as to whether adopting video glasses for other studies with IAPS picture material might yield higher emotional responses than do the usual screen presentations. Typical findings of studies in which IAPS slides 
are employed include increased arousal ratings and electrodermal activity for unpleasant and pleasant pictures in comparison with neutral pictures. Pleasure ratings show monotonic increases from unpleasant to neutral to pleasant pictures. Heart rate (HR) also predominantly shows a valence modulation, with greater deceleration during the viewing of unpleasant slides than during the viewing of neutral or pleasant slides. ${ }^{1}$ These effects were found for short (6-sec) presentations in mixed designs, in which picture valence alternated within a single presentation block (see, e.g., Bradley, Cuthbert, \& Lang, 1990; Greenwald, Cook, \& Lang, 1989; Lang, Greenwald, Bradley, \& Hamm, 1993), as well as in designs with presentation blocks of pictures of homogeneous valence (Bradley, Cuthbert, \& Lang, 1996). An interesting development for the valence modulation of HR states that this cardiac pattern emerges only when participants have no prior information about the valence categories that are to be viewed (Bradley, 1998). If they are cued by prior information or growing experience in sustained or repeated experimental designs, then HR would be modulated by arousal with decelerations for both pleasant and unpleasant materials in comparison with neutral slides. Thus, it should also be found for extended presentations of series with a block design, which are required for addressing specific research questions, as was discussed by Sutton, Davidson, Donzella, Irwin, and Dottl (1997).

In the present study, we compared the use of 2-D video glasses and a video projector in the prolonged presentation of three valence-grouped IAPS series in 24 female participants. In response to previous suggestions, a withinsubjects design with measurements on 2 separate days was employed, because psychophysiological measures demonstrate large individual differences (Ravaja, 2004). We hypothesized that, due to the focused attention, reduced distraction, and enhanced presence of the visual material, the video glasses prompt stronger responses in affective reports and autonomic reactions as quantified by skin conductance level (SCL) and HR. We further expected an arousal modulation of HR with stronger deceleration for both the pleasant and the unpleasant series than for the neutral series.

\section{METHOD}

\section{Participants}

Twenty-four healthy female students (average age $=27.88$ years, $S E=1.21$ years) were recruited for this study. Undergraduate students received credit points for participation. The data of an initial test with 6 participants were excluded because of partial equipment failure. In compliance with the instructions of the video glasses manufacturer, epileptic symptoms were an exclusion criterion. Informed consent was obtained from all the participants.

\section{Materials}

Ninety pictures were extracted from the IAPS (Center for the Study of Emotion and Attention, 1999) and grouped into pleasant, neutral, and unpleasant affective categories with 30 pictures in each series. The selection was based on normative ratings for valence and arousal offered in the technical manual of the IAPS (Lang, Bradley, \& Cuthbert, 1997). All three series began with a 30 -sec baseline while a monochrome slide was shown. Pictures were presented for $10 \mathrm{sec}$ each without interstimulus intervals, resulting in presentation times of $5 \mathrm{~min}$ for each of the three series. To prevent rapid habituation to the series, the pictures within each series were shown in reverse order on the 2 nd presentation day. All of the series were preprogrammed as timed computer presentations on a notebook (Satellite 2140 CDS, Toshiba, Tokyo), from which they were transferred to the 2-D video glasses (Eye-Trek FMD-700, Olympus, Tokyo) and the projector (VPL-PX32, Sony, Tokyo). The resulting size of all colored pictures was adjusted to be approximately the same for both modalities - that is, a 52-in. monitor impression from a 2-m distance.

\section{Measures}

Affective ratings. The subjects rated their experienced pleasure (valence) and arousal for each series with a paper-and-pencil version of the 9-point Self-Assessment Manikin (SAM; Hodes, Cook, \& Lang, 1985).

Physiological measures. SCL (in $\mu$ siemens) was recorded under a constant voltage of $0.4 \mathrm{~V}$ using a sampling rate of $50 \mathrm{~Hz}$. The Ag$\mathrm{AgCl}$ electrodes (9 $\mathrm{mm}$ in diameter) were filled with electrolyte gel (Sigma Crème, Parker Laboratories, Fairfield, NJ) and attached to the distal phalanx of the ring finger and little finger of the nondominant hand. HR (in bpm) was calculated offline from R-R intervals in the recorded electrocardiogram, which was measured with a sampling rate of $200 \mathrm{~Hz}$. Ag-AgCl electrodes (55 $\mathrm{mm}$ in diameter) were placed on the insides of the left ankle and right wrist with a reference to the right ankle (Einthoven II). SCL and HR were simultaneously recorded using a PAR-PORT/F module (PAR Medizintechnik, Berlin).

\section{Procedure}

After a short introduction, all the electrodes were attached and the room lights were dimmed. Sitting in a recliner, the participants were familiarized with the procedure and the SAM. A 5-min resting period followed to facilitate laboratory adaptation. After the final instructions were given, the pleasant, neutral, and unpleasant 5-min series were presented, always preceded by a $30-\mathrm{sec}$ prestimulus baseline and with 2-min intervals between series. The affective ratings, given immediately after each series, consisted of asking each participant about her emotional experience during the entire series. Twelve of the participants were randomly assigned to view the series with video glasses on the 1st day and with the projector on the 2 nd day, 1 week later. The other 12 were assigned to the reverse order. The six possible presentation orders of the three series were counterbalanced across all participants but remained the same on both days for each individual. The subjects were informed prior to the experimental sessions that pleasant, neutral, and unpleasant series would be presented.

\section{Data Analysis}

HR and SCL were averaged across the complete 30 -sec prestimulus baselines and across the complete 5-min epoch of each picture series for both presentation modes. Means for prestimulus baselines were subtracted from the averaged 5-min epoch of the respective series. Difference scores $(\triangle \mathrm{HR}, \Delta \mathrm{SCL})$ and pleasure and arousal ratings were analyzed as dependent variables in repeated measures ANOVAs with 3 (affective category: pleasant vs. neutral vs. unpleasant) $\times 2$ (presentation mode: video glasses vs. projector) $\times 2$ (mode order: video glasses on 1st day and projector on 2 nd day vs. projector on 1st day and video glasses on 2 nd day) levels. Polynomial a priori contrasts were performed by testing the hypothesized quadratic trends for main effects in arousal ratings, $\triangle \mathrm{SCL}$, and, following Bradley (1998), $\Delta \mathrm{HR}$ as well, with higher values for the affective series in comparison with the neutral series. A monotonic trend with 
ratings increasing from the negative to the neutral to the positive series was expected for pleasure ratings. In addition, explorative between-subjects ANOVAs were conducted for all measures acquired with the video glasses in comparison with those acquired with the projector, separately for the first and second presentation days. These contained 3 (affective category) $\times 2$ (presentation mode) levels. Stronger responses for the video glasses were expected to show significant interactions for affective category $\times$ presentation mode in the ANOVAs, followed by significant univariate pairwise comparisons (video glasses $>$ projector) calculated for each of the three affective categories. Furthermore, a repeated measures ANOVA was calculated for averaged SCL with 3 (affective category) $\times 2$ (presentation mode) $\times 2$ (measurement time: baseline vs. 5 -min epoch) levels. This was chosen to examine possible SCL decreases over the course of time that have been reported in earlier studies (Bradley et al., 1996). Newman-Keuls post hoc tests $(p<.05)$ were performed to explore unexpected significant interactions obtained in the ANOVAs. A Greenhouse-Geisser correction was applied in cases in which sphericity assumptions were violated.

\section{RESULTS}

\section{Affective Ratings}

The picture series showed the expected overall effects on pleasure and arousal ratings, with highly significant effects for affective category $[F(2,44)=125.74, p<.001$, $\varepsilon=.78$, and $F(2,44)=64.11, p<.001$, for the pleasure ratings and the arousal ratings, respectively]. Pleasure ratings showed a significant monotonic increase from the unpleasant to the neutral to the pleasant series $[F(1,22)=$ 163.77, $p<.001]$, whereas a quadratic trend revealed higher arousal for the pleasant and unpleasant series in comparison with the neutral series $[F(1,22)=93.96, p<$ .001 ; Figure 1, upper panels]. Pleasure ratings showed no significant interaction effect for affective category $X$ presentation mode, but did show a significant interaction of affective category $\times$ presentation mode $\times$ mode $\operatorname{order}[F(2,44)=3.83, p<.05]$. For the unpleasant series, post hoc tests revealed significantly more unpleasantness for the projector presentation on the 1st day than for the video glasses presentation on the 2 nd day. In contrast, although not significantly so, for the unpleasant series the video glasses presentation on the 1st day prompted higher unpleasantness ratings than did the projector presentation on the 2 nd day. These findings suggest a habituation effect between both sessions.

For arousal ratings, a significant interaction was found for affective category $\times$ presentation mode $[F(2,44)=$ $5.76, p<.01]$. Post hoc analyses showed the expected higher ratings for the video glasses mode than for the projector mode for the pleasant series $[F(1,23)=3.02, p<$ $.05]$, but unexpected higher arousal ratings for the projector mode after the unpleasant series $[F(1,23)=5.23$, $p<.05]$. Moreover, a significant interaction of presentation mode $\times$ mode order for arousal ratings was found
(A) Pleasure

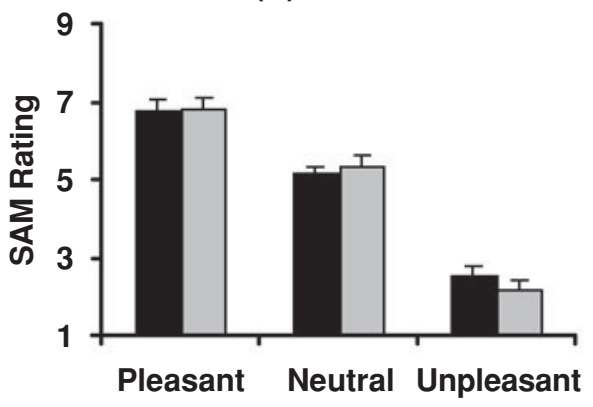

(C) Heart Rate Change

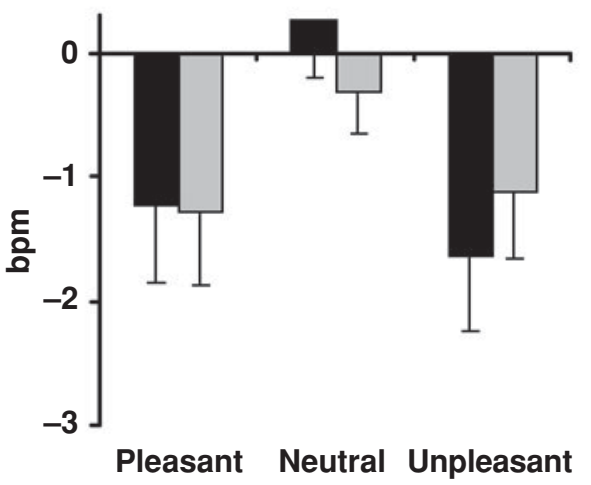

(B) Arousal

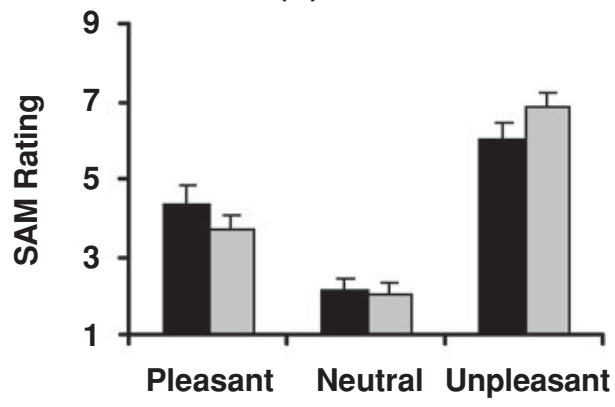

(D) Skin Conductance Change

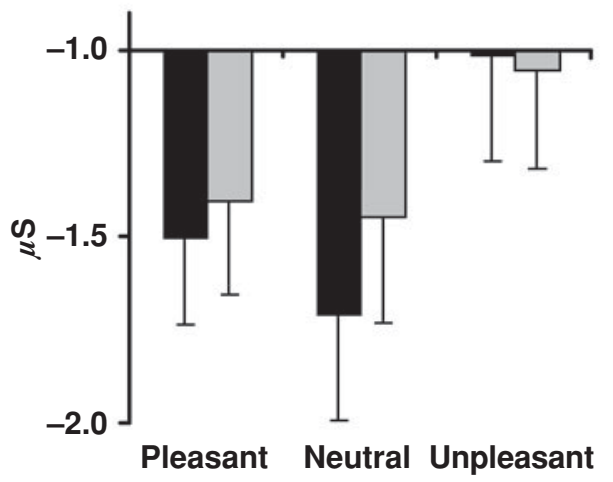

Figure 1. Pleasure, arousal, heart rate change, and skin conductance change for the pleasant, neutral, and unpleasant picture series for video glasses (black bars) and projector (gray bars). Error bars represent standard errors of the means. 
$[F(1,22)=10.73, p<.01]$. Post hoc tests showed nonsignificant trends $(p \mathrm{~s} \leq .08)$ for higher averaged ratings on the 1 st presentation day in comparison with the 2nd day, irrespective of presentation mode. These main effects of order indicated a habituation effect for arousal ratings.

\section{Physiological Measures}

Values for autonomic measures are displayed in Figure 1 (lower panels). For $\Delta \mathrm{HR}$, a significant effect of affective category was found $[F(2,44)=4.05, p<.05]$. A quadratic trend revealed the expected decelerations for both the unpleasant and the pleasant series in comparison with the neutral series $[F(1,22)=10.29, p<.01]$. No significant interactions were found.

A significant effect of affective category was found for SCL as well $[F(2,44)=3.30, p<.05]$. A marginal quadratic trend showed the expected higher response rates for both the unpleasant and the pleasant series in comparison with the neutral series $[F(1,22)=2.91, p<.10]$. Across all series in both presentation modes, a significant decrement in averaged SCL over time was obtained in comparison with the relevant baselines $[F(1,23)=57.39$, $p<.001]$. A significant interaction was found for affective category $\times$ mode order $[F(2,44)=4.18, p<.05]$. Post hoc analyses showed that this unexpected effect was due to weaker electrodermal activity in the pleasant series in comparison with the unpleasant series for the presentation mode of projector before video glasses. Moreover, a significant interaction for affective category $\times$ presentation mode was found for $\triangle \mathrm{SCL}$ in the explorative betweensubjects ANOVA for the 1st presentation day $[F(2,44)=$ $3.19, p=.05]$. Post hoc analyses showed higher electrodermal activity during presentation of the pleasant series with video glasses in comparison with its presentation in the projector mode $[F(1,23)=3.63, p<.05]$. No other significant interactions were obtained.

\section{DISCUSSION}

The results of this study demonstrate that video glasses are an effective instrument for presenting prolonged affective picture series. This converges with the findings of the first study in which this presentation mode was used within the picture viewing paradigm (von Leupoldt \& Dahme, 2005). Nonetheless, the expected general superiority of this new instrument in eliciting emotional responses, in comparison with commonly employed screen presentations by video projector, could not be shown. Instead, both modes emerged as equally capable of manipulating affective states in a sustained design, as is shown by the expected main effects across both modalities. Therefore, the application of video glasses might be preferable only in specific laboratory settings associated with limited view to ordinary screens and monitors. For example, studies requiring extensive and constricting measuring devices located close to the participant's head and visual field might benefit from this presentation mode. In particular, studies in which respiratory responses to affective stimulation are recorded by means of complex breathing circuits, face masks, or nose clips could profit from the use of video glasses, as has already been shown (von Leupoldt \& Dahme, 2005). The known distracting effects on emotion induction associated with specific pulmonary measuring instruments placed in the face area might thus be significantly reduced by focusing the attention on the presented visual material (Ritz, Dahme, et al., 2002; von Leupoldt \& Dahme, 2004). Furthermore, video glasses can also be used appropriately for stimulus presentations in the limited space of fMRI or PET scanners. Therefore, it must be assured that all magnetic details of the glasses are replaced by nonmagnetic materials. Another application of this technique could be in studies with immobilized participants, which have to be conducted in uncontrolled nonlaboratory settings - for example, private homes or hospital rooms. Comparable and nondistractive settings could thus be created by presenting the required tasks or stimuli with video glasses.

However, the main effects of the present study showed a valence modulation for pleasure ratings, whereas an arousal modulation was obtained for arousal ratings and electrodermal activity with higher responses for the affective series in comparison with the neutral series. These results with a prolonged block design are in line with many other studies in which the picture viewing paradigm with a mixed design was used (e.g., Bradley et al., 1990; Greenwald et al., 1989; Lang et al., 1993; Winton, Putnam, \& Krauss, 1984). However, a hypothesized arousal modulation was also observed for HR. These findings for the cardiac pattern converge with findings reported by Bradley (1998) and discussed by Ritz, Alatupa, et al. (2002) for respiratory changes. Following this lead, an arousal modulation for HR can be expected rather than a valence modulation, if participants are familiar with the affective range of the pictorial stimuli to be viewed.

In general, our hypotheses regarding the superiority of video glasses over projector presentations were not confirmed. Instead, only two measures tended to confirm the expected effects. First, the arousal reports after the pleasant picture series showed the hypothesized higher ratings for video glasses in comparison with the projector. This effect was mirrored by higher electrodermal activity during the pleasant series when it was presented with video glasses, in comparison with the projector presentation, on the 1st day. We can only speculate on the exclusive effect of these arousal measures during the pleasant emotional condition. As some participants stated, this was due to the fact that the experience of greater privacy and intimacy with video glasses led to stronger involvement, especially during viewing of erotic slides predominantly depicting nude couples and nude males. Thus, the obtained increases in arousal ratings and SCL converge with previously reported effects of enhanced presence due to new or differ- 
ent presentation media (Lombard \& Ditton, 1997; Reeves, Lang, Kim, \& Tatar, 1999). These presumed increases of involvement and perceived realism of the pictures could, however, be influenced by other variables, such as image size (Reeves et al., 1999). Although the image size was adjusted to be comparable between video glasses and projector, it was not systematically manipulated in the present study, which limits our ability to draw a definite conclusion about this effect.

However, for unpleasant picture series the opposite effect (i.e., higher arousal reports for the projector presentation) was found. Here, the technical features of a typical laboratory environment could have been more apparent during the open view to the screen, which might have amplified the arousing character of the unpleasant slides in individual participants. The marginally lower pleasure ratings for the unpleasant series in the projector mode are slightly indicative of this idea. Alternatively, it might be argued that the participants were not equally attentive during the video glasses presentation. This point of criticism cannot be fully dismissed, since no manipulation check by measurement of eye or lid movements could be carried out due to interferences between the video glasses and the required measuring devices, were these to be adjacently located. However, when questioned after the presentation with video glasses, all the participants stated that they followed the instructions and fully concentrated on the pictures. Thus, reduced attention to the picture series in the video glasses mode seems to be a less likely explanation for the present findings in the arousal ratings. However, in general the pleasure ratings did not support the findings for arousal reports, since no significant difference between the presentation modes was obtained.

Although not consistently significant, several effects or interactions for affective category, presentation mode, and/or mode order are indicative of habituation between the 2 experimental days. This was found for pleasure and arousal ratings and also appeared as a trend for HR. Stronger responses were shown for the 1st day and first presentation mode in comparison with the 2 nd day and second presentation mode. Thus, our results correspond with reported decreases in physiological reactivity after repeated picture presentations (Bradley, Lang, \& Cuthbert, 1993; Sutton et al., 1997).

The fact that we focused only on female participants might be seen as a limitation of our study. Although no general sex differences in the experience of the video glasses were hypothesized, stronger electrodermal reactivity in males as well as higher arousal ratings for pleasant pictures have been reported (Bradley \& Lang, 2000; Greenwald et al., 1989; Lang et al., 1993). Further validation of the presented method should therefore include a male population. At variance with previous suggestions (Ravaja, 2004), a between-subjects design might be more appropriate for this specific experimental context with regard to the obtained habituation effects between the successive presentation days.

\section{REFERENCES}

Bentsen, B., Svensson, P., \& Wenzel, A. (1999). The effect of a new type of video glasses on the perceived intensity of pain and unpleasantness evoked by a cold pressor test. Anesthesia Progress, 46, 113-117.

Berenson, A. B., Wiemann, C. M., \& Rickert, V. I. (1998). Use of video eyeglasses to decrease anxiety among children undergoing genital examinations. American Journal of Obstetrics \& Gynecology, 178, 1341-1345.

Bradley, M. M. (1998). Assessing the heart's desire: Cardiac patterns in affective perception [Abstract]. Psychophysiology, 35, 21.

Bradley, M. M., Cuthbert, B. N., \& Lang, P. J. (1990). Startle reflex modification: Emotion or attention? Psychophysiology, 27, 513-522.

Bradley, M. M., Cuthbert, B. N., \& Lang, P. J. (1996). Picture media and emotion: Effects of a sustained affective context. Psychophysiology, 33, 662-670.

Bradley, M. M., \& LANG, P. J. (2000). Measuring emotion: Behavior, feeling, and physiology. In R. Lane \& L. Nadel (Eds.), Cognitive neuroscience of emotion (pp. 242-276). New York: Oxford University Press.

Bradley, M. M., Lang, P. J., \& Cuthbert, B. N. (1993). Emotion, novelty, and the startle reflex: Habituation in humans. Behavioral Neuroscience, 107, 970-980.

CEnTER For the Study of Emotion And AtTEntion (1999). The International Affective Picture System: Digitized photographs. Gainesville: University of Florida, Center for Research in Psychophysiology.

Frere, C. L., Crout, R., Yorty, J., \& McNeil, D. W. (2001). Effects of audiovisual distraction during dental prophylaxis. Journal of the American Dental Association, 132, 1031-1038.

Greenwald, M. K., Cook, E. W., \& Lang, P. J. (1989). Affective judgment and psychophysiological response: Dimensional covariation in the evaluation of pictorial stimuli. Journal of Psychophysiology, 3, 51-64.

Hamm, A. O., \& Vaitl, D. E. (1993). Emotionsinduktion durch visuelle Reize: Validierung einer Stimulationsmethode auf drei Reaktionsebenen. Psychologische Rundschau, 44, 143-161.

Hodes, R. L., Cook, E. W., III, \& LANG, P. J. (1985). Individual differences in autonomic response: Conditioned association or conditioned fear? Psychophysiology, 22, 545-557.

LANG, P. J., Bradley, M. M., \& CuthBert, B. N. (1997). International Affective Picture System (IAPS): Technical manual and affective ratings. Gainesville: University of Florida.

Lang, P. J., Bradley, M. M., \& Cuthbert, B. N. (1998). Emotion and motivation: Measuring affective perception. Journal of Clinical Neurophysiology, 15, 397-408.

Lang, P. J., Greenwald, M. K., Bradley, M. M., \& Hamm, A. O. (1993). Looking at pictures: Affective, facial, visceral, and behavioral reactions. Psychophysiology, 30, 261-273.

Lombard, M., \& DitTon, T. (1997). At the heart of it all: The concept of presence. Journal of Computer-Mediated Communication, 3(2). Retrieved January 21, 2006 from jcmc.indiana.edu/vol3/issue2/ lombard.html.

RAVAJA, N. (2004). Contributions of psychophysiology to media research: Review and recommendations. Media Psychology, 6, 193-235.

Reeves, B., Lang, A., Kim, E. Y., \& TAtar, D. (1999). The effects of screen size and message content on attention and arousal. Media Psychology, 1, 49-67.

Ritz, T., Alatupa, S., Thöns, M., \& Dahme, B. (2002). Effects of affective picture viewing and imagery on respiratory resistance in nonasthmatic individuals. Psychophysiology, 39, 86-94.

Ritz, T., Dahme, B., Dubois, A. B., Folgering, H., Fritz, G. K., HarVER, A., ET AL. (2002). Guidelines for mechanical lung function measurements in psychophysiology. Psychophysiology, 39, 546-567.

Ritz, T., George, C., \& Dahme, B. (2000). Respiratory resistance during emotional stimulation: Evidence for a nonspecific effect of experienced arousal? Biological Psychology, 52, 143-160.

Sutton, S. K., Davidson, R. J., Donzella, B., Irwin, W., \& Dottl, D. A. (1997). Manipulating affective state using extended picture presentations. Psychophysiology, 34, 217-226.

von LeupoldT, A., \& DAHME, B. (2004). Emotions in a body plethys- 
mograph: Impact of affective film clips on airway resistance. Journal of Psychophysiology, 18, 170-179.

von Leupoldt, A., \& DAhMe, B. (2005). Emotions and airway resistance in asthma: Study with whole body plethysmography. Psychophysiology, 42, 92-97.

Winton, W. M., Putnam, L. E., \& Krauss, R. M. (1984). Facial and autonomic manifestations of the dimensional structure of emotion. Journal of Experimental Social Psychology, 20, 195-216.

\section{NOTE}

1. Other measures have also been found to show strong associations with the dimensions of arousal (e.g., cortical slow wave, viewing time, interest ratings) and valence (e.g., corrugator and zygomatic muscle activity, startle blink reflex), but because these have not been a subject of this study they are not discussed further. For an overview, see Bradley and Lang (2000) or Lang, Bradley, and Cuthbert (1998).

(Manuscript received June 5, 2004;

revision accepted for publication December 2, 2004.) 